\title{
The Effect of the Type of HA on the Degradation of PLGA/HA Composites
}

Ashutosh Naik*a, David V. Shepherda, Jennifer H. Shepherda, Serena M. Besta and Ruth E. Cameron ${ }^{\mathrm{a}}$

a Cambridge Centre for Medical Materials, University of Cambridge, Department of Materials Science and Metallurgy, 27, Charles Babbage Road, Cambridge, CB3 OFS, $U K$

\section{Abstract:}

The aim of this study was to explore the importance of the potentially competing effects of buffering effects of the calcium phosphate filler and particle-mediated water sorption on the degradation products of Poly (D, L lactide-co-glycolide (50:50))(PLGA)/ Hydroxyapatite(HA) composites . Further the influence of type of HA on the mechanical properties of the composites was investigated. Phase pure HA was synthesised via a reaction between aqueous solutions of calcium hydroxide and orthophosphoric acid. The powder produced was either used as produced (uncalcined) or calcined in air or calcined in a humidified argon atmosphere. An in-vitro degradation study was carried out in phosphate buffered saline (PBS). The results obtained indicated that the degradation rate of the composite might be better understood if both the buffering effects and the rate of water sorption by the composites are considered.

* Coressponding Author: Ashutosh Naik Email - naikashutosh @gmail.com ; Keywords: Hydroxyapatite; Poly (D, L lactide-co-glycolide (50:50)); buffering effects; particle-mediated water sorption 


\section{Abbreviations:}

HA, Hydroxyapatite; PLGA, poly (D, L lactide-co-glycolide (50:50)); PBS, Phosphate buffered saline; X-Ray diffraction, XRD; ICP-AES, Inductively coupled plasma- Atomic emission spectroscopy;

\subsection{Introduction:}

Synthetic biodegradable polymers have been used as medical materials since the late 1960s when the first biodegradable polymer suture material was approved (Dexon $\left.{ }^{\circledR}\right)($ Schmitt and Frazza,1971). Poly $\alpha$-hydroxy acids such as polyglycolide, polylactide and their co-polymers are some of the most common polymers used. These polymers are attractive materials for medical applications such as coatings for medical devices,(Stubbe et. al.,2004) tissue engineering(Douglas et. al.,2009,Porter et. al.,2009) and drug delivery(Cartiera et. al.,2009, Silva de Melo et. al.,2012).

HA belongs to a family of compounds known as apatites that closely resemble the mineral component of bone. In order to mimic natural bone, a composite at the nanoscale (Rho et. al.,1998),HA is often used as filler in bone analogue composites.

One of the main functions of biodegradable composites is to provide support during the natural healing process of bone. The composites must not only stimulate cellular function in the designated time frame but must also transfer the load from the surrounding bone to regenerating tissue to encourage new 
bone formation(Tanck et. al.,2006). The design of composites with the necessary degree of functional complexity relies on understanding the degradation properties of the material after implantation. Many synthetic aliphatic polyesters degrade mainly by chemical hydrolysis of the polymer backbone(Vert et. al.,1992). One of the ways of controlling the hydrolysis that takes place within the polymer is through the addition of alkaline calcium phosphates to the polymer. Calcium phosphates dissolve and partially neutralise the acidic local environment (Ehrenfried et al.,2008; Yang et al.,2009). This reduces the autocatalysis in the sample and slows the degradation of the material as a whole. However, the addition of calcium phosphate particles may also change the degree of hydration of the composite (Lin et al.,1999, Li and Chang,2005) and hence the concentration of reactants in the degradation reaction. These competing effects of buffering effects of the calcium phosphate filler and particlemediated water sorption may influence the degradation of the composites.

In the study presented in this paper, PLGA 30-wt\% HA composites are produced using three different types of HA as filler and an in-vitro degradation study carried out in phosphate buffered saline (PBS) to explore the interplay of these effects. Mechanics of the composites are also investigated.

\subsection{Materials and Methods:}




\subsection{Materials}

The materials were used as-received unless stated otherwise. Calcium hydroxide $\left(\mathrm{Ca}(\mathrm{OH})_{2}, 99+\%\right)$ was purchased from Fisher Scientific, UK and orthophosphoric acid $\left(\mathrm{H}_{3} \mathrm{PO}_{4}, 85 \%\right)$ was purchased from Acros Organics, UK. PLGA (50:50) was purchased from Surmodics Pharmaceuticals, USA. PBS (0.01M) powder was purchased from Sigma Aldrich, UK. Standard solutions used for the detection of calcium concentration were purchased from Fisher Scientific, UK.

\subsection{HA synthesis}

HA was synthesised using a wet chemical method. Aqueous solutions of $0.5 \mathrm{M}$ $\mathrm{Ca}(\mathrm{OH})_{2}$ and $0.3 \mathrm{M} \mathrm{H}_{3} \mathrm{PO}_{4}$ were used as reactants. A pH>10.5 was maintained with ammonia addition at room temperature. The HA thus synthesised was ground, milled and sieved using a $180 \mu \mathrm{m}$ sieve. The powder was used as produced and will be referred to as "uncalcined HA." In addition the powder was calcined at $800^{\circ} \mathrm{C}$ for 4 hours in either an atmosphere of air or wet argon and will be referred to as "HA calcined in air" and "HA calcined in wet" respectively.

\subsection{X-Ray Diffraction (XRD)}

XRD was carried out in order to characterise the phase composition and crystallinity of HA powder. The machine used was a Philips PW 1830 diffractometer with $\mathrm{Cu} \mathrm{K}$ radiation. The run was carried out at $2 \theta=10-50^{\circ}$ with a step size of $0.05^{\circ}$ and dwell time of $8 \mathrm{~s}$. Divergence and anti-scatter slits of $0.5^{\circ}$ and a receiving slit of $0.2 \mathrm{~mm}$ were used. 


\subsection{Composite production}

A two-step process produced the composites; the first involved the mixing of the polymer and filler in acetone; the second, injection moulding of the mixture.

\subsubsection{Mixing}

PLGA and 30-wt\%HA were mixed in a PTFE container with acetone as solvent for 1 hour using an overhead magnetic stirrer. The mix was then allowed to stand for a further hour before drying in a vacuum oven at $40^{\circ} \mathrm{C}$ overnight.

\subsubsection{Injection Moulding}

The mixture of PLGA and HA was processed into dumbbell shaped specimens (to be used for studying elastic mechanical properties), and cylindrical specimens (for the degradation study and to study plastic deformation) using a $12 \mathrm{~cm}^{3} \mathrm{DSM}$ Xplore mini injection moulder. A mould temperature of $35^{\circ} \mathrm{C}$ and melt temperature of $140^{\circ} \mathrm{C}$ was used. The injection pressure was 5.0 bar whereas the filling pressure and the holding pressure were 2.0 bar. The holding time for the uncalcined sample was $3 \mathrm{~s}$ for each step, however for the calcined samples the holding time was changed to $6 \mathrm{~s}$ in order to allow the formation of complete specimens.

\subsection{Characterisation of composites}

\subsubsection{Particle size of filler}

A JEOL 5800 in back scattered mode at an accelerating voltage of $10 \mathrm{kV}$ was used to observe the surfaces of the composites and in order to calculate the particle size of fillers within the composites. The freeze fractured surfaces of the samples 
were prepared by immersing the dumbbell shaped specimens in liquid nitrogen and cutting from the gauge length region using a wire cutter.

\subsubsection{Filler content of composites}

In order to compare the weight fraction of HA present in the composite to the intended (30wt\%) weight fraction a Q500 TGA was used. The process to measure mass loss with temperature increase was carried out in an atmosphere of nitrogen.

Approximately 20-30 mg of sample was used. The samples were cut from the centre as well as the edges of the cylinders produced using the method described earlier. The temperature was increased at a rate of $40^{\circ} \mathrm{C} / \mathrm{min}$ from room temperature to $500^{\circ} \mathrm{C}$. Three repeats were carried out for each sample.

\subsection{In-vitro degradation study}

In order to assess how the type of HA influences the degradation of the composites, hydrolytic degradation at $37^{\circ} \mathrm{C}(\mathrm{pH}=7.4)$ was carried out in PBS (0.01 M) dissolved in $1 \mathrm{~L}$ de-ionised water. The cylindrical specimens were cut into discs ( $2 \mathrm{~mm} \times 2 \mathrm{~mm})$ using a Struers Accutom 5 . 
The samples once cut were weighed individually and the correct amount of PBS added in a ratio of $6 \mathrm{mg} / \mathrm{ml}$ (sample/PBS). Each sample was tied in unbleached muslin and suspended in the PBS. After the addition of the PBS, the samples were maintained at $37^{\circ} \mathrm{C}$ without further agitation. The PBS was unchanged during the period of degradation. Pure polymer was used as a control. Twenty-four time points were studied in total and three replicates were made for each time point. The results presented are an average of the three repeats. However, a total of nine $\mathrm{pH}$ readings were taken for the samples from day 7 to day 45 and six readings for the day 1 and day 50 samples.

The degradation of the composites was characterised by measuring: water absorption, mass loss, and calcium concentration in solution and change in $\mathrm{pH}$.

\subsubsection{Water absorption}

The mass of the wet samples ( $\left.\mathrm{M}_{\text {wet }}\right)$ at each time point was measured after dabbing it with a paper towel on removal from the PBS in order to absorb the surface water. The samples were dried using a vacuum oven at $40^{\circ} \mathrm{C}$ and a pressure of 800 mbar for 24 hours. The samples were then weighed to measure the dry mass of the samples $\left(\mathrm{M}_{\mathrm{dry}}\right)$.

The water absorption $\left(\mathrm{M}_{\mathrm{w}}\right)$ of the samples was calculated using Eq (1)

$M_{w}=\left(\left(M_{\text {wet }}-M_{\text {dry }}\right) / M_{\text {dry }}\right) * 100 \%$ 


\subsubsection{Mass Loss}

The samples were weighed before immersion in PBS ( $\left.\mathrm{M}_{\text {Initial}}\right)$ and after drying (M dry). The samples were measured using an electronic balance (AG204 Mettler-Toledo, UK) with an accuracy of $0.1 \mathrm{mg}$. The mass loss $\left(\mathrm{M}_{\mathrm{L}}\right)$ was calculated using Eq (2)

$\mathrm{M}_{\mathrm{L}}=\left(\left(\mathrm{M}_{\text {dry }}-\mathrm{M}_{\text {Initial }}\right) / \mathrm{M}_{\text {Initial }}\right) * 100 \%$

\subsubsection{Calcium concentration in solution}

The calcium concentration in the PBS after composite degradation was determined using a Liberty Ax Sequential ICP-AES (Varian Inc.). The machine was calibrated using the standard solutions (1 ppm, 10 ppm, 100 ppm, 1000 ppm) for calcium and approximately $5 \mathrm{ml}$ of sample used in each run. Stock PBS solution was used as a control. 


\subsubsection{Change in $\mathrm{pH}$}

The $\mathrm{pH}$ of the solution was measured during the degradation using an electronic bench-top pH meter (Orion 3 Star, Thermo Electron Corporation). Before each set of measurements, the $\mathrm{pH}$ meter was calibrated using standard solutions.

\subsection{Mechanical Properties}

\subsubsection{Elastic properties}

DMTA (DMA Q800, TA Instruments, USA) was used to measure the viscoelastic properties of the injection- moulded samples. The dumbbell shaped specimens used were $35 \mathrm{~mm}$, in length, $4.1 \mathrm{~mm}$ in width and $2.1 \mathrm{~mm}$ in thickness. The tests were carried out in cantilever mode using a dual cantilever clamp. A multi frequency module was used at four different frequencies: 1, 10, 20 and $50 \mathrm{~Hz}$. The tests were carried out between $-10^{\circ} \mathrm{C}$ to $120^{\circ} \mathrm{C}$, with a heating rate of $3^{\circ} \mathrm{C} / \mathrm{min}$. Three repeats were carried out for each sample. The results shown are for the tests carried out at $1 \mathrm{~Hz}$.

\subsubsection{Plastic deformation}

A Hounsfield (5 kN) machine was used to carry out compression tests on the composites. The cylindrical shaped specimens with dimensions of $12 \mathrm{~mm} \times 6$ 
mm were used as samples. A total of three repeats were carried out for each sample.

\subsection{Results:}

\subsection{Characterisation of powders}

Fig.1 shows the XRD spectra obtained for the synthesised HA powders. The scans were compared with reference ICDD spectra for hydroxyapatite, the red lines indicating the position of the reference peaks (09-432). The uncalcined HA powder exhibited broad peaks with, in some cases relatively low intensity. Sharper peaks with higher intensity are observed compared with those of the uncalcined HA powder. The aluminum peak seen in the scan for the calcined in air HA was due to the stub used, but otherwise no impurity phases were observed.

\subsection{Characterisation of composite}

Table 1 shows a comparison of the particle size (in terms of mean particle size ) of the HA in the composite and the HA powders. The mean size of particles was smaller when the powders were used as fillers for the composites.

Table 2 shows that the weight fraction of HA obtained from different regions of the samples were similar to the intended weight fraction despite the difference in the filler used. 


\subsection{In-vitro degradation study}

The degradation study was carried out for 60 days. The samples of pure polymer had degraded by day 21 and the analyses for mass loss and water absorption were only possible up to day 14 because by day 21 the polymer samples were fully degraded.

\subsubsection{Water Absorption and Mass Loss}

The water absorption and mass loss observed up to day 21 are shown in Fig.2. The data was very noisy beyond this point and no clear trends were observed.

The pure PLGA samples in this study showed lowest water absorption between day $1(2.6 \pm 0.4 \%)$ and day $14(15.4 \pm 3.3 \%)$ and the samples could not be analysed beyond this point as they had fully degraded by day 21(Fig.2).

The composites containing HA absorbed more water than the pure polymer samples but no clear trends were observed in the water absorption characteristics for the composite containing calcined (air or wet) HA powder $(62.2 \pm 13.1$ and $63.0 \pm 9.0$ respectively at day 21$)$ or uncalcined HA (49.6 \pm 5.8$)$ during the degradation study (Fig.2).

The pure polymer could not be analysed beyond day 14 ((-5.3 $\pm 5.2 \%)$, Fig.2). The negative sign indicates mass loss. Similar mass loss was recorded for the composites containing calcined HA up to day $21(-12.2( \pm 7.9) \%$ and $-7.0( \pm 1.5) \%)$, which was lower than the mass loss observed for the composite containing uncalcined HA (-27.3( \pm 2.9$) \%)$ (Fig.2). However, no clear difference was noted in 
the mass loss observed for the composite containing calcined in air HA and the composite containing calcined in wet HA.

\subsubsection{Calcium concentration in solution:}

The composite containing uncalcined HA had released the greatest amount of calcium up to day 35 . The amount of calcium released in ppm was about $69 \mathrm{ppm}$ at day 21 rising to $174 \mathrm{ppm}$ by day 35 . By comparison the calcium concentration was 13 ppm for the composites containing calcined HA at day 21 (Fig.3).

\subsubsection{Change in $\mathrm{pH}$ :}

The $\mathrm{pH}$ values observed for all samples between day 0 and day 21 were similar and certainly until day 14 remained largely constant at close to neutral $\mathrm{pH}$ (Fig.4).

The $\mathrm{pH}$ profile for the samples containing 30-wt\% HA is, as expected, different from that of pure polymer (as seen at day 21 in Fig. 4). The composite containing uncalcined HA showed a sharp drop in pH from day 21 to day $28(6.1( \pm 0.2)$ $4.6( \pm 0.3))$.

The $\mathrm{pH}$ profile for the composites containing calcined HA was different from that obtained for the composite containing uncalcined HA. The $\mathrm{pH}$ readings at day 21 for the composites containing calcined HA were closer to $7(6.9( \pm 0.1)$ for the composite containing calcined in air $\mathrm{HA}$ and $6.9( \pm 0.1)$ for the composite 
containing calcined in wet HA) compared to $6.1( \pm 0.2)$ for the composite containing uncalcined HA.

\subsection{Mechanical properties}

\subsubsection{Elastic mechanical properties}

Fig. 5 shows the average storage modulus ( $\left.\mathrm{E}^{\prime}\right)$ of samples at $20^{\circ} \mathrm{C}$. The $\mathrm{E}^{\prime}$ values for the composites containing calcined HA (calcined in wet $=5.8( \pm 0.5) \mathrm{GPa}$ calcined in air $=6.3( \pm 0.5) \mathrm{GPa}$ ) were higher than those for the composite containing uncalcined HA $(3.1( \pm 0.3) \mathrm{GPa})$. The E' values for the composites were higher than the corresponding values for pure polymer $(2.6( \pm 0.5) \mathrm{GPa})$.

\subsubsection{Plastic mechanical properties}

The lowest value for UTS obtained was 64.4( \pm 2.7$) \mathrm{MPa}$ for the composite containing uncalcined HA whereas those for the composite containing calcined in air HA and the composite containing calcined in wet HA were $69.6( \pm 1.9) \mathrm{MPa}$ and 88.3( \pm 1.5$)$ MPa respectively (Fig. 6).

An increase of over $35 \%$ was observed for the composites containing calcined in wet HA (88.3 \pm 1.5$)$ when compared with the composite containing uncalcined HA (64.4 \pm 2.7$)$. The increase was not as pronounced for the composite containing

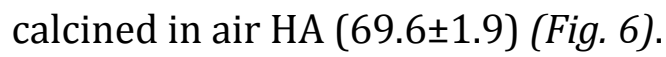

\subsection{Discussion:}

\subsection{Composite structure:}

The method of composite production used may have resulted in a reduction in the agglomeration of the filler within the composite compared with powders alone and hence a smaller mean diameter was observed (Table 1). 
When the weight fraction of the filler is $\mathbf{5} 30$-wt $\%$ in PLGA/CaP composites, negligible agglomeration has been reported. However, beyond 30-wt\% (40 and 50-wt\%) a high degree of agglomeration and non-uniform distribution of filler particles was observed(Hosseinabadi et. al.,2011). These large agglomerates can reduce particle/polymer interaction and prevent load transfer(Jose et. al.,2009). PLGA/30wt\% HA composites were produced using different fillers (uncalcined, calcined in air and wet HA). The weight fraction of HA obtained from different regions of the samples was similar to the intended weight fraction below the value at which agglomeration might be expected for each filler used. Therefore, differences in the mechanical properties and degradation profiles of the composites obtained maybe attributed to the difference in filler characteristics.

\subsection{Water sorption:}

When a homogeneous polymer is placed in an aqueous medium, water penetrates the polymer leading to cleavage of the ester bonds(Li S 2008, Ali et. al.,1993, Schmitt et. al.,1994). The rate of polymer hydrolysis has been reported in literature to be independent of the total amount of water absorbed by the polymer, but dependent on the amount of bound water. Solid-state ${ }^{2} \mathrm{H}$ NMR has been used to demonstrate the presence of two types of water within the polymer bulk water with free rotation and bound water with hindered rotation. The hydrolysis rate was reported to be dependent on the amount of water hydrogen bound to the oxygen of ester carbonyl groups(Schmitt et. al.,1994). The 
degradation rate of poly ( $\alpha$-hydroxy acids) is also influenced by their residual monomer content (Park et. al.,1994).

The addition of calcium phosphate filler has been reported by the same authors to increase the amount of water absorbed by the composites during the initial stages of degradation (Naik A et.al., 2015), which could be due to the hydrophilicity of the calcium phosphate (Wang et. al.,2011). However, in contrast, other authors report a decrease in the water uptake of composites containing calcium phosphate filler(Lin et. al.,1999).

In this case, the decrease in water uptake was attributed to the blocking of water by the filler particles preventing the entry of water into the interior of the polymer (Lin et. al., 1999). The results obtained in the present study agree with the trend observed by Naik A et.al., 2015 as the percentage of water absorbed by the composites up to day 21 was greater than that of pure polymer (Fig.2).

\subsection{Mass Loss:}

The mass loss observed during degradation of the composite can be linked to the pH of the buffer solution(Li et. al., b 1990). It has been reported that weight loss associated with polymer degradation in buffer solutions occurs in three stages. In the first stage there is low weight loss with a rapid decrease of molecular weight. In the second stage, severe weight loss starts along with slowing down of molecular weight loss and the formation of monomers. In the final stage, weight loss is total. About half the polymers are converted to monomers with 
subsequent hydrolysis and the formation of lactic acid and glycolic acid (Hakkarainen et. al.,1996).

The degradation of PLGA is a combination of homogeneous as well as heterogeneous degradation( $L i$ et. al., ${ }^{b}$ 1990, Li et.al., ${ }^{a}$ 1990). Initially water penetrates the samples and hydrolytic cleavage of ester bonds and production of carboxylic end groups leads to homogeneous degradation of PLGA. The autocatalytic effect of the acidic end groups created by the scission of polymeric chains along with the restricted diffusion of oligomers from the inner parts of the sample are the important factors leading to heterogeneous degradation ( $\mathrm{Li}$ et. al., b1990, , Li et.al., ${ }^{a} 1990$, Grizzi et. al.,1995, Hurrell and Cameron,2001).

The faster degradation of the pure polymer when compared to the composites is likely to be due to the enhanced rate of hydrolytic scission of the ester bonds arising from the autocatalysis of the polymer(Li et. al., ${ }^{b} 1990$, Li et.al., $^{a} 1990$, Ginde and Gupta,1987). The presence of calcium phosphate decreases the rate of degradation by neutralizing the acidic environment thereby reducing autocatalysis (Ehrenfried et. al.,2008, Yang et. al.,2009).

At least part of the enhanced mass loss in the composite containing uncalcined HA over the composites containing calcined HA particles, maybe accounted for by the faster dissolution of uncalcined HA particles, as reflected in the higher calcium concentration obtained for the composite containing uncalcined HA at day 21(Fig.3). Uncalcined HA is less ordered and hence expected to be more soluble than the calcined HA particles. It has been reported that dissolution of filler particles creates pores of similar dimensions on the surface of composites that are likely to facilitate the diffusion of water in the composite and hence 
diffusion of acidic by-products from the inner part of the sample thereby accelerating degradation (Niemela T, 2005)resulting in lowering the $\mathrm{pH}$ of the solution despite the buffering effect of the filler.

\subsection{Calcium concentration:}

The calcium concentration obtained from the ICP represents the extent of dissolution of HA. The calcium concentration obtained for the composite containing uncalcined HA at day 21 was the highest among the composites analysed The results obtained for the composite containing calcined in wet HA indicated less dissolution of the calcined in wet HA powder compared with the other two fillers, as proposed by Christoffersen et al., (1998). This is one possible explanation for a slower degradation profile.

\section{$4.5 \mathrm{pH}$ :}

The $\mathrm{pH}$ values observed for all samples up to day 21 were similar and certainly until day 14 remained largely constant at close to neutral $\mathrm{pH}$. The $\mathrm{pH}$ profile for the samples containing 30-wt\% HA is, as expected, different from that of pure polymer. As indicated the HA neutralizes the carboxylic end groups released by polymer degradation (as seen in Fig.4). The composite containing uncalcined HA also showed a sharp drop in pH from day 21 to day $28((6.1( \pm 0.2)-4.6( \pm 0.3))$. Fig.4).

The $\mathrm{pH}$ profile for the composites containing calcined HA were different from that obtained for the composite containing uncalcined HA. The $\mathrm{pH}$ readings at day 21 for the composites containing calcined HA were closer to 7. In addition the $\mathrm{pH}$ drop seen in the composite containing uncalcined HA is not as drastic for the composites containing calcined HA. 
4.6 Effect of buffering and water sorption:

The results presented in this paper indicate the importance of two competing effects (buffering effect of filler and the effect of hydration) on the degradation profile of the composites.

The buffering effect of the filler, results in lowering the degradation rate of the composite. Fig.7 (a) shows a schematic for the buffering effect that the type of filler might be expected to have on the degradation profile of the composites produced for this study if this was the only influencing variable on degradation. The addition of $\mathrm{HA}$ as filler delays the degradation and also alters the degradation rate of the composite compared with the pure polymer sample. The degree of delay depends on the type of HA used as filler, and the composite containing uncalcined HA as filler would result in the highest degree of delay due to faster dissolution of uncalcined HA (resulting in higher pH of the solution) when compared with the composites containing calcined HA powders. The shaded region represents the time point during the degradation study when the type of filler no longer influences the degradation rate of the composite.

The effect of hydration (amount of water absorbed), might be expected to result in an increase in the degradation rate of the composite, if there is an associated increase in the amount of bound water (Schmitt et. al.,1994) . Fig.7 (b) shows a schematic for the expected effect of increased bound water (considered independently) on the degradation profile of the composites produced for this 
study. The pure polymer sample would exhibit the longest degradation times since the addition of calcium phosphate resulted in an increase in water absorption. The higher amount of water absorbed would then be expected to lead to faster degradation.

During a degradation study however, the two competing effects influence the rate of degradation for the composites simultaneously. Fig.7(c) shows the schematic for the simultaneous effects of type of buffer and hydration on the composites produced in this study. The schematic indicates that the degradation rate of the composite can be better understood if both the buffering effects on autocatalysis and the rate of water absorbed by the composites are considered.

\section{Mechanical properties:}

The values for $\mathrm{E}^{\prime}$ obtained at $20^{\circ} \mathrm{C}$ for the composites containing HA reported in this paper (Fig.5) are from well within the glassy region that there is a lack of chain mobility within the polymer which is further restricted due to the stiffening effect of the filler(Zhao et. al.,2012) .

The ultimate tensile strength (UTS) for composites depends on the reinforcement of the polymer by filler and increases with increase in filler(Lee et.al.,2008, Jeong et.al.,2008). In addition the UTS depends on the interfacial adhesion between polymer and filler(Jeong et.al.,2008, Liu and Webster, 2010). Fig. 6 shows the UTS values obtained for the composites containing HA. The observed increase in UTS in the composites made from calcined in wet HA maybe attributed to the $\mathrm{OH}$ groups retained on the surface of these particles. 


\subsection{Conclusions:}

An in-vitro degradation study of PLGA-30wt\% HA composite was carried out in PBS. The use of HA significantly altered the degradation profile of the composite compared to the pure polymer. This effect was particularly seen when calcined in wet powder was used as the filler during the early part of the degradation study. The mechanical properties (elastic and plastic) of composites containing calcined HA were higher than those for uncalcined HA and pure polymer. The results showed the importance of the two competing effects of buffering effect of filler which delays the rate of degradation and particle-mediated water sorption which results in increasing the rate of degradation of the composites. The degradation rate of the composite can be better understood if both the buffering effects on autocatalysis and the rate of water absorbed by the composites are considered. These considerations are relevant to the rational design of resorbable composites for applications in orthopaedic medicine and given the considerable improvement in the degradation profile and the demonstrable significant improvement in the mechanical properties a further study is warranted.

The desirability of the research cannot be underscored further. 
Tables and Figures:

Table 1: Mean size of HA particles (in $\mu \mathrm{m}$ ) within composite and as powder

\begin{tabular}{|l|l|l|}
\hline Sample Name & Powder $(\mathbf{d}(\mathbf{0 . 5}))($ in $\boldsymbol{\mu m})$ & Composite $(\mathbf{d}(\mathbf{0 . 5}))($ in $\boldsymbol{\mu m})$ \\
\hline Uncalcined HA & 10.6 & 4.9 \\
\hline Calcined in air HA & 8.3 & 4.5 \\
\hline Calcined in wet HA & 6.0 & 4.1 \\
\hline
\end{tabular}

Table 2: : Average value of filler content for unmodified HA composites

\begin{tabular}{|l|l|}
\hline Sample Name & Weight fraction of filler \%( \pm S.E.) \\
\hline Uncalcined HA & $27.7( \pm 0.4)$ \\
\hline Calcined in air & $29.5( \pm 0.6)$ \\
\hline Calcined in wet & $31.2( \pm 0.1)$ \\
\hline
\end{tabular}


Characterisation of HA:

XRD Spectra for synthesised HA powders

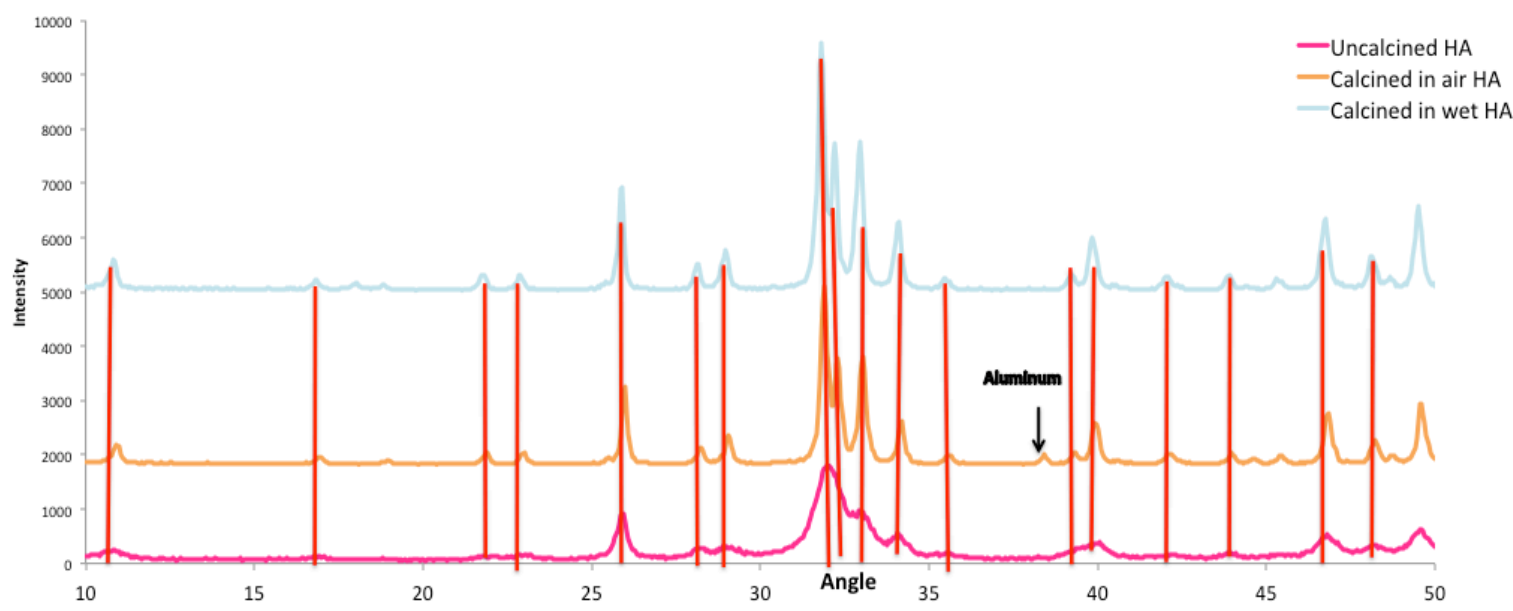

Fig.1: XRD spectra for synthesised HA powders 
Degradation study:

Water absorption and mass loss: up to Day 21

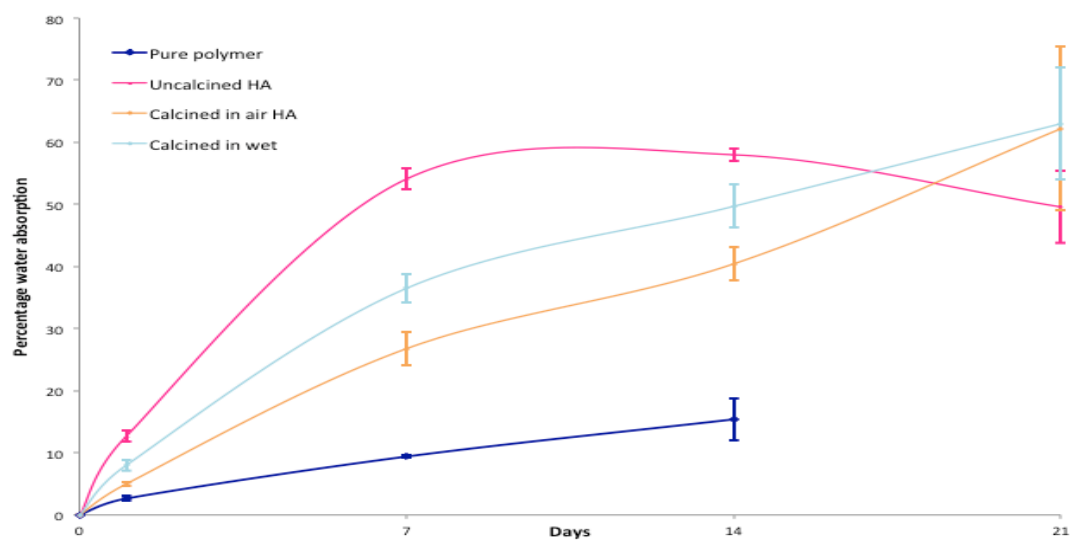

(a)

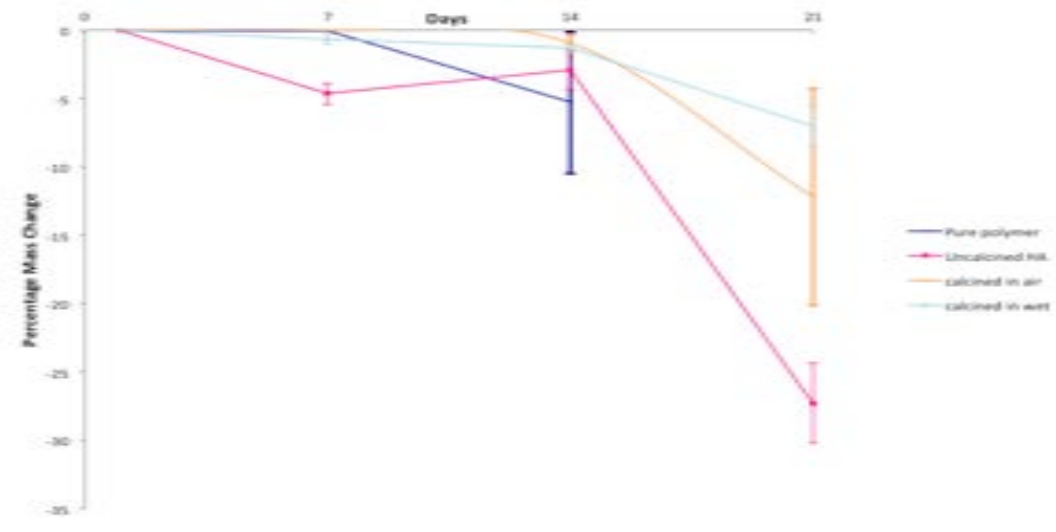

(b)

Fig.2: (a) Water Absorption and (b) Mass Loss up to day 21 
Calcium concentration in solution:

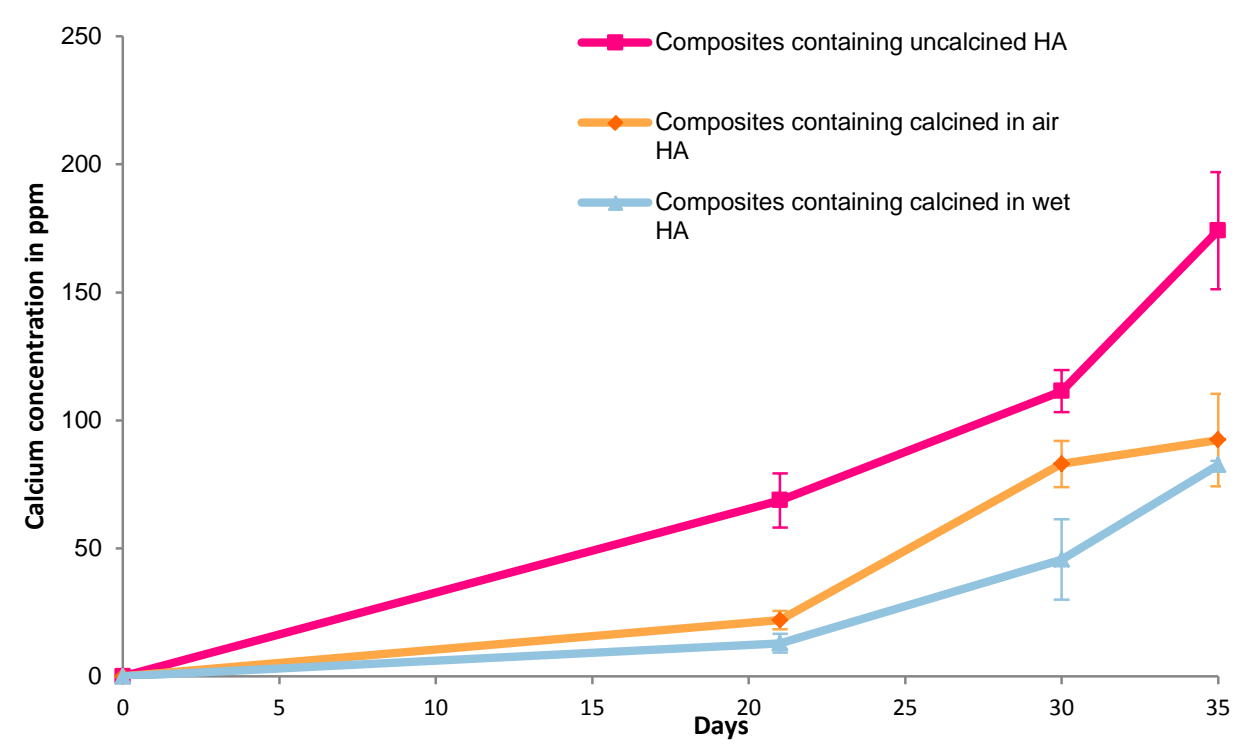

Fig.3: Calcium concentration in solution for composites containing HA

Change in $\mathrm{pH}$ of solution:

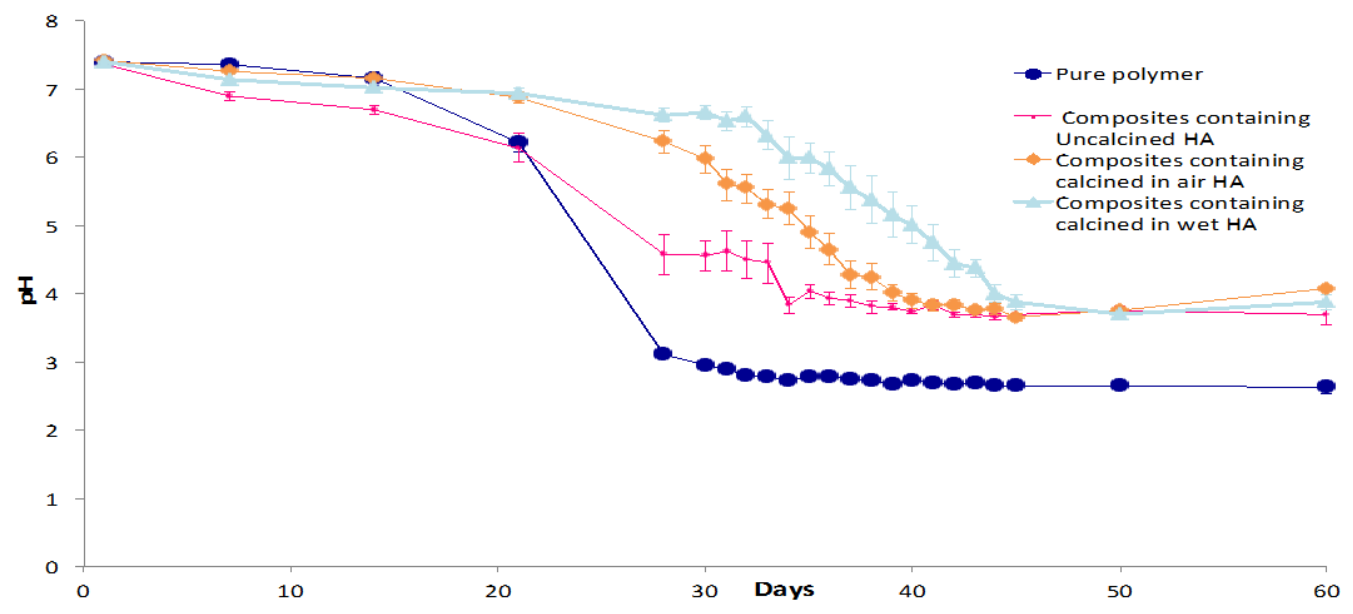

Fig.4: Change in pH of solution 
Elastic mechanical properties:

Storage Modulus (E') of samples at $20^{\circ} \mathrm{C}$

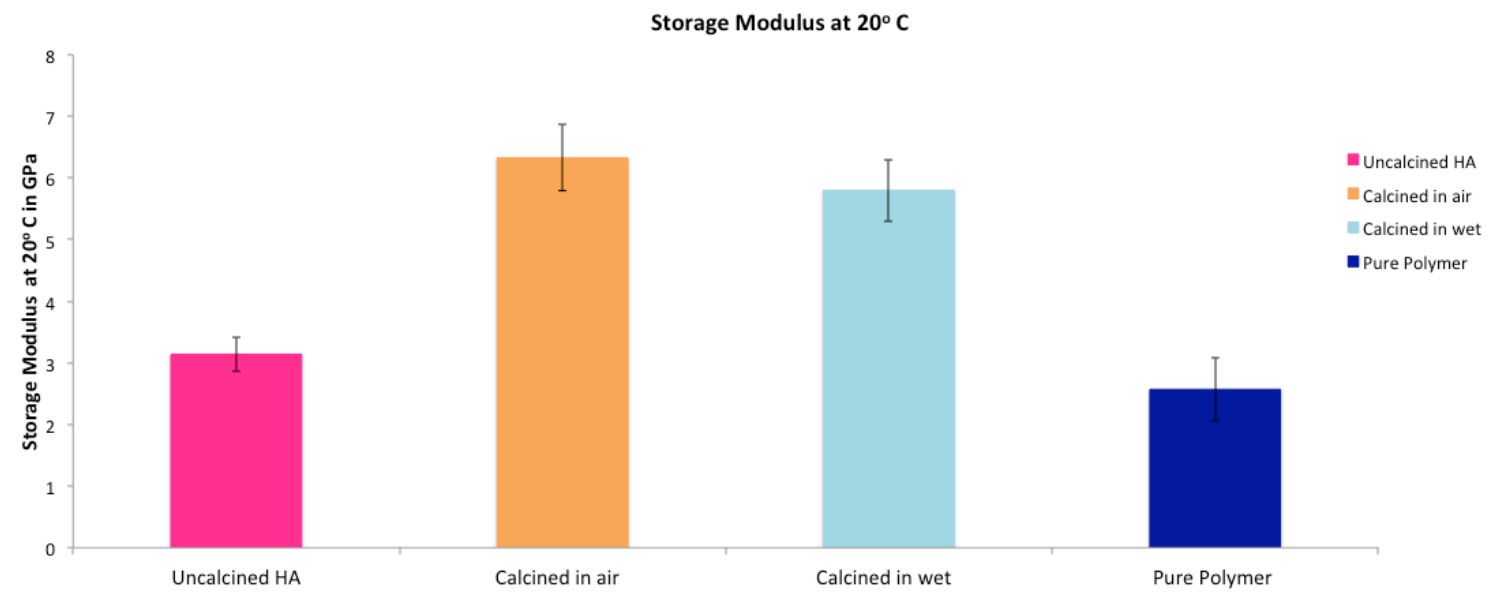

Fig.5: Storage Modulus (E') of samples at $20^{\circ} \mathrm{C}$

Plastic mechanical properties:

UTS at room temperature

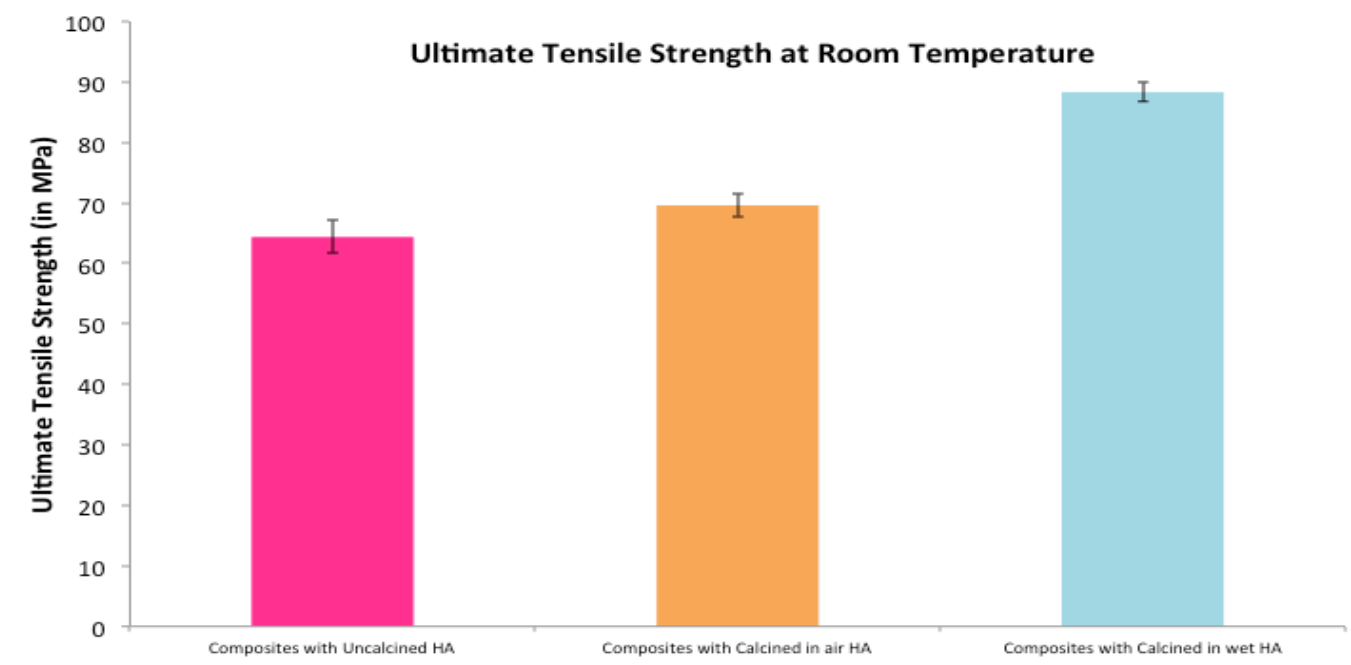

Fig.6: Plastic mechanical properties UTS of composites containing HA at room temperature 
Schematic of competing effects:
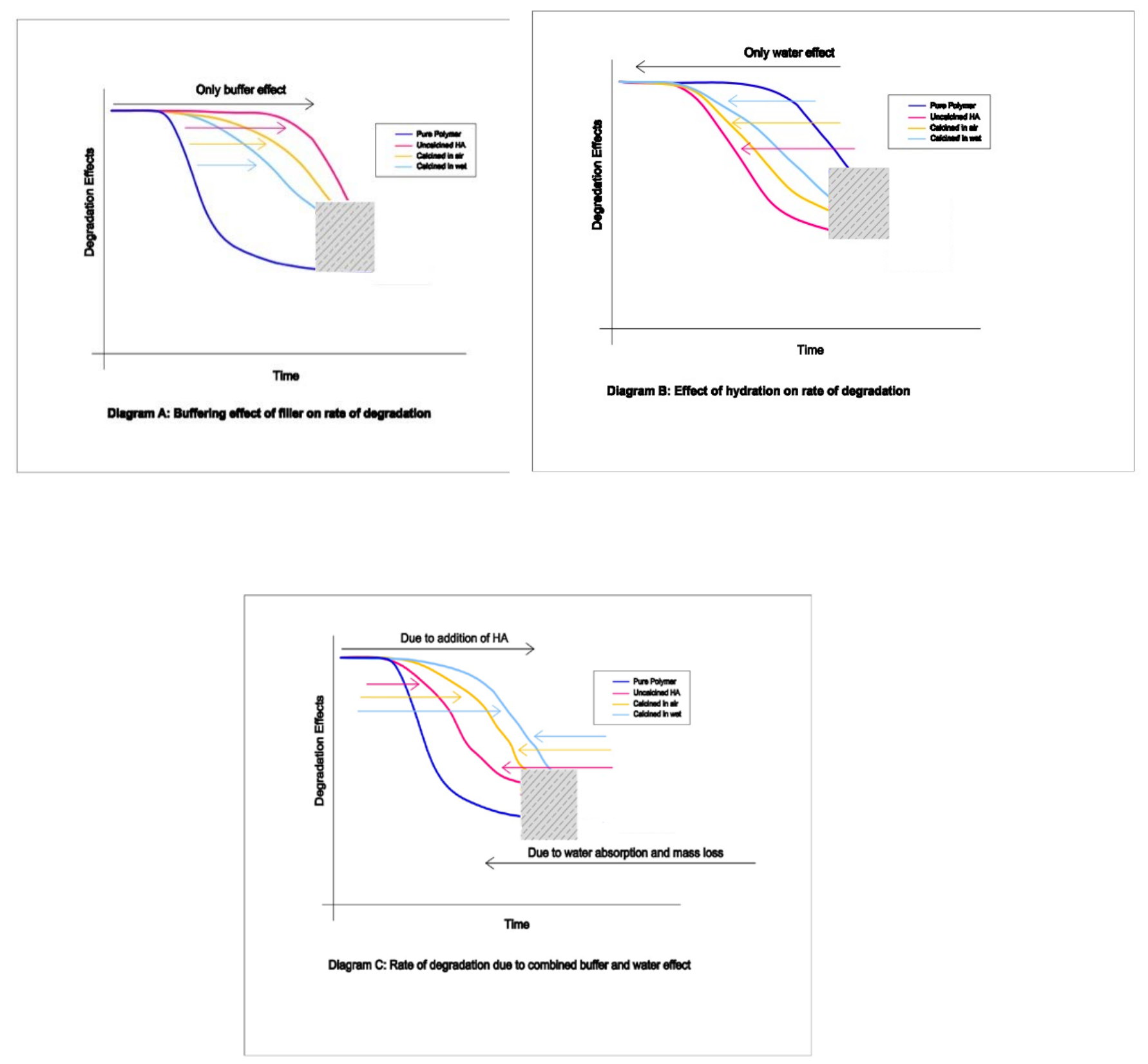

Fig. 7: (a) Schematic showing the effect of filler (buffering effect) on the rate of degradation (b) Schematic showing the effect of hydration (water absorbed) on the rate of degradation (c) Schematic showing the simultaneous effect of filler and hydration on degradation rate of composite 


\section{References:}

Ali S, Doherty PJ, Williams DF. "Mechanisms of polymer degradation in implantable devices. 2. Poly (DL lactic acid)." Journal of Biomedical Materials Research. 27; 1409-18 (1993).

Cartiera, M.S., Johnson, K.M., Rajendran, V., Caplan, M.J., and Saltzman, W.M “ The uptake and intracellular fate of PLGA nanoparticles in epithelial cells." Biomaterials._30; 2790-98(2009).

Douglas T, Pamula, E., Hauk, D., Wiltfang, J, Sivananthan, S, Sherry E, and Warnke PH "Porous polymer/hydroxyapatite scaffolds: characterization and biocompatibility investigations". Journal of Materials Science: Materials in Medicine. 20; 1909-15(2009).

Ehrenfried LM, Patel MH, Cameron RE “The effect of tri-calcium phosphate (TCP) addition on the degradation of polylactide-co-glycolide (PLGA)." Journal of Materials Science: Materials in Medicine 19:459-66(2008).

Ginde RM and Gupta RK "In vitro chemical degradation of poly (glycolic acid) pellets and fibres". Journal of Applied Polymer Science 33; 241129(1987).

Grizzi I, Garreau H, Li S, Vert M. (1995)."Hydrolytic degradation of devices based on poly (DL-lactic acid) size dependence". Biomaterials 16(4); 30511(1995).

Hakkarainen M, Albertson A, Karlsson S “Weight loss and molecular changes correlated with the evolution of hydroxyacids in simulated in-vivo degradation of homo and co polymers of PLA and PGA". Polymer Degradation and Stability 52; 283-91(1996). 
Hosseinabadi ME, Ashrafizadeh F, Etemadifar M, Venkatraman SS. "Preparation and mechanical behavior of PLGA/nano-BCP composite scaffolds during in-vitro degradation for bone tissue engineering." Polymer Degradation and Stability.96; 1940-6 (2011).

Hurrell S and Cameron."Polyglycolide: degradation and drug release. Part I: Changes in morphology during degradation". Journal of Materials Science Materials in Medicine 12; 811-16(2001).

Jeong SI, Ko EK, Yum JS, Jung CH, Lee YM, Shin H "Nanofibrous poly (lactic acid)/Hydroxyapatite composite scaffolds for guided tissue regeneration". Macromolecular Bioscience. 8; 328-38(2008).

Jose MV, Thomas V, Johnson KT, Dean DR, Nyairo E . “Aligned PLGA/HA nanofibrous nanocomposite scaffolds for bone tissue engineering." Acta Biomaterialia 5 305-15 (2009) .

Lee JB, Lee SH, Yu SM, Park JC, Choic JB, Kim JK "PLGA scaffold incorporated with hydroxyapatite for cartilage generation." Surface and coatings Technology. 202; 5757-61 (2008).

Li H and Chang J "pH-compensation effect of bioactive inorganic fillers on the degradation of PLGA" Composites Science and Technology 65 2226$32(2005)$.

Li S In vitro physicochemical test methods to evaluate bioresorbability. Degradation rate of bioresorbable materials. Prediction and evaluation. Cambridge Woodhead Publishing. Fraser Buchanan(2008).

Li, S.M., Garreau, H. Vert, M. "Structure property relationships in the case of the degradation of massive aliphatic poly ( $\alpha$-hydroxy acids) in aqueous- 
media, part 1: poly (DL-lactic acid). Journal of Materials Science-Materials in Medicine 1; 123 -130(1990a).

Li, S.M., Garreau, H., and Vert, M. "Structure property relationships in the case of the degradation of massive aliphatic poly ( $\alpha$-hydroxy acids) in aqueousmedia.2: degradation of lactide-glycolide copolymers - PLA37.5GA25 and PLA75GA25." Journal of Materials Science-Materials in Medicine 1; 131$39\left(1990^{b}\right)$.

Lin FH Chen TM, Lin CP and Lee CJ "The merit of Sintered PDLLA/TCP composites in management of bone fracture internal fixation." Artificial Organs. 23(2); 186-94(1999).

Liu $H$ and Webster TJ "Mechanical properties of dispersed ceramic nanoparticles in polymer composites for orthopedic applications." International Journal of Nanomedicine_5; 299-313(2010).

Naik, A., Best, S. M., \& Cameron, R. E. (2015). “The influence of silanisation on the mechanical and degradation behaviour of PLGA/HA composites." Materials Science and Engineering: C, 48, 642-650.

Niemela $\mathbf{T}$ "Effect of $\beta$-tricalcium phosphate addition on the in vitro degradation of self-reinforced poly-L,D-lactide." Polymer Degradation and Stability 89; 492-500 (2005).

Park TG "Degradation of poly (D,L-lactic acid) microspheres: effect of molecular weight." Journal of Controlled Release 30; 161-173(1994).

Porter JR, Henson A. and Popat, KC." Biodegradable poly (epsiloncaprolactone) nanowires for bone tissue engineering applications". Biomaterials. 30; 780-88(2009). 
Rho JY, Kuhn-Spearing L, Zioupos P."Mechanical properties and the hierarchical structure of bone". Medical engineering and physics 20; 92-102 (1998).

Schmitt EA, Flanagan DR, Linhardt RJ "Importance of distinct water environments in the hydrolysis of poly (DL-Lactide-co-glycolide)." Macromolecules 27; 743-48(1994).

Schmitt EE and Frazza EJ "A new absorbable suture". Journal of Biomedical Materials Research Symposium. 1; 43-58(1971).

Silva de Melo NF, Ribiero de Araujo D, Grillo R, Morales Moraes C Prado de Matos A, De Paula E, Rosa AH, Fraceto LF "Benzocaine-loaded polymeric nanocapsules: Study of anaesthetic activities". Journal of Pharmaceutical Sciences 101(3); 1157-65(2012).

Stubbe BG, De Smedt SC and Demeester J"Programmed polymeric devices for pulsed drug delivery”. Pharmaceutical Research_21; 1732-40(2004).

Tanck E, Hannink G, Ruimerman R, Buma P, Burger EH, Huiskes R" Cortical bone development under the growth plate is regulated by mechanical load transfer". Journal of Anatomy.208;73-79(2006).

Vert M, Li SM, Spenlehauer G Guerin P “Bioresorbability and biocompatibility of aliphatic polyesters". Journal of Materials Science : Materials in Medicine 3; 432-46(1992).

Wang T, Chow LC, Frukthbeyn SA, Ting HA, Dong Q, Yang M, Mitchell JW"Improve the Strength of PLA/HA Composite Through the Use of Surface Initiated Polymerization and Phosphonic Acid Coupling Agent". Journal of Research of National Institute of Standards and Technology 116(5): 785796(2011). 
Yang Z, Best SM, and Cameron RE “The Influence of a-Tricalcium Phosphate Nanoparticles and Microparticles on the Degradation of Poly (D,L-lactide-coglycolide)." Advanced Materials 21; 3900-04(2009).

Zhao Z, Shan W Zhang Y, Li X, Ma J Yan Y "Fabrication and Properties of Degradable Poly(amino acid)/Nano Hydroxyapatite Bioactive Composite." Journal of Applied Polymer Science 125; 2502-09(2012). 